\title{
Decisions on pricing, capacity investment, and introduction timing of new product generations in a durable-good monopoly
}

\author{
Richard F. Hartl ${ }^{1}$ (D) Peter M. Kort ${ }^{2,3} \cdot$ Andrea Seidl $^{1}$ \\ Published online: 6 November 2019 \\ (c) The Author(s) 2019
}

\begin{abstract}
The aim of the present paper is to analyze how firms that sell durable goods should optimally combine continuous-time operational level planning with discrete decision making. In particular, a firm has to continuously adapt its capacity investments and sales strategy, but only at certain times it will introduce a new version of the durable good to the market. The launch of a new generation of the product attracts new customers. However, in order to be able to produce the new version, production facilities need to be adapted leading to a decrease of available production capacities. We find that the price of a given generation of a product decreases over time. A firm should increase its production capacity most upon introduction of a new product. The stock of potential consumers is largest then so that the market is most profitable. The extent to which existing capacity can still be used in the production process for the next generation has a non-monotonic effect on the time when a new version of the product is introduced as well as on the capital stock level at that time.
\end{abstract}

Keywords Durable goods · Introduction of new product generation · Capacity investments

$凶$ Richard F. Hartl

richard.hartl@univie.ac.at

1 Department of Business Decisions and Analytics, University of Vienna, Oskar-Morgenstern-Platz 1, 1090 Vienna, Austria

2 Department of Econometrics and Operations Research \& CentER, Tilburg University, P.O. Box 90153, 5000 LE Tilburg, The Netherlands

3 Department of Economics, University of Antwerp, Prinsstraat 13, 2000 Antwerp, Belgium 


\section{Introduction}

This paper is about the optimization problem of a firm that sequentially introduces different versions of a durable good on the market. "A durable good is a good that does not quickly wear out, or more specifically, one that yields utility over time rather than being completely consumed in one use. Highly durable goods such as refrigerators, cars, or mobile phones usually continue to be useful for three or more years of use, so durable goods are typically characterized by long periods between successive purchases." 1

Our aim is to find out how firms that sell durable goods, combine continuous-time operational-level planning (continuously deciding on capacity investment and sales) with discrete strategic decision making (when to launch a new generation of the durable product). The practical applications we have in mind are, e.g., when does Samsung replace its Galaxy S9 by S10, or when does Apple introduce a new generation of the iPhone.

Concerning the policy of setting an output price, essentially two basic strategies can be observed in practice. First, for a given product version the price is always kept constant. The main advantage of such a strategy (employed e.g. by Apple) is that the consumers do not have an incentive to wait with purchasing the product until the price has dropped sufficiently. The other strategy (e.g. applied by Samsung) is that the price of a given product can be varied over time. This enabled to employ a more tailor made policy with respect to serving different types of consumers. In particular one can distinguish between early adopters that are less price sensitive and more price conscious consumers who intend to wait until the given product has a lower price. The first policy of a constant price was already analyzed in Seidl et al. (2019). The present paper modifies and extends that work by analyzing a firm that could vary the price over time.

The so-called Coase conjecture, see Coase (1972), states that a monopolist has to sell the durable good at a price equal to its marginal costs. The reasoning behind this conjecture is that consumers are aware that a firm has to lower its price if they do not purchase the product for a given price. However, in practice, this outcome is hardly ever observed. Consider for example the price of a smart phone which is usually the highest when the phone is launched and decreases over time. In the present paper we confirm that it is optimal to employ such a strategy and discuss how the heterogenous willingness of the consumers to pay for a product as well as capacity restrictions affect the price.

The papers McAfee and Wiseman (2008) and Montez (2013) analyze situations in which the outcome of the Coase conjecture (does not) occur in a single product generation problem. The pricing of two generations of a durable product is discussed for example in Dhebar (1994), Kornish (2001) and Li and Graves (2012). Şeref et al. (2016) consider the introduction and the pricing of two generations in a diffusion model. We extend literature by analyzing the impact of increasing the number of generations and find that the actual optimal number of generations is infinite.

\footnotetext{
${ }^{1}$ See https://en.wikipedia.org/wiki/Durable_good.
} 
How the diffusion of a durable product is affected by constrained production capacity in a make-to-stock production is investigated by Ho et al. $(2002,2011)$ in a single generation problem. The impact of the price in such a framework is considered in Shen et al. (2014). Note that a more extensive literature review can be found in Seidl et al. (2019).

In the present paper, we investigate in a rather simple model how the interaction of pricing and capacity investments is affected by the possibility of introducing new generations of a product. To thoroughly understand the implications of multiple product generations we start by analyzing the problem with a fixed number of generations which we increase gradually. It is shown that the actual optimal number of product generations is infinite, which is reasonable due to the assumption that each generation only has a limited total number of potential consumers and because the planning period is infinite.

Concerning the optimal investment strategy it is found that in the beginning of each product generation the investments into capacity are the largest. Then one also charges the highest price to compensate for these investments. The optimal price decreases over time as one tries to win consumers who are not able or willing to purchase the product for a high price.

The impact of different parameters is analyzed. In particular, we find that the market potential, the costs for introducing a new product generation and the extent to which one can reuse capacity for the subsequent generation of the product can have some nontrivial effect on the optimal solution. We compare the results obtained in the present paper, where the price follows from the inverse demand function, to the results of Seidl et al. (2019), where the optimally determined price was assumed to be constant. We find that the non-constant price allows higher sales of any product generation, thus, at least for some time investments into capacity are also higher. As a consequence of the possibility to attract more consumers, the switching time to the next product generation increases.

The present model shares some similarities to vintage capital stock models where also multiple generations of a product are considered. In our model, a certain product generation appears at an optimally determined time, whereas in a vintage capital stock model this holds for a certain vintage of the capital stock. If each new vintage would produce a new generation of a durable good, the models are equivalent.

The present paper was chosen by the authors for this special issue celebrating the scientific achievements of Gustav Feichtinger in recognition of his enthusiasm and work in the area of multi-stage modeling, see e.g. Bultmann et al. (2008), Grass et al. (2008), Caulkins et al. (2011b, 2013a,b), Moser et al. (2014) and Caulkins et al. (2015), and product innovation, see e.g. Feichtinger et al. $(2004,2006)$ and Caulkins et al. (2007, 2011a).

The paper is organized as follows. The model is presented in Sect. 2, whereas Sect. 3 contains the analysis. Section 4 concludes. 


\section{The model}

There are two state variables, namely $Q$, which is the stock of potential consumers for the durable good, and $K$, which is the firm's production capacity. Each consumer buys one item at most. Once the item is bought, the consumer disappears from the market. The continuous control variables are $q$, which is the quantity of the good sold to the consumers and $I$, the capacity investment. We have production costs, $c q$, and investment costs $I+\frac{\theta}{2} I^{2}$.

Further we have $p(Q, q)$, which is the inverse demand function, where

$$
p(Q, 0)>0, \quad \frac{\partial p(Q, q)}{\partial q}<0, \quad \frac{\partial p(Q, q)}{\partial Q}>0 .
$$

It makes sense that the reservation price is higher if the number of potential consumers is larger. We assume here a linear relationship in that the reservation price equals $f(Q)=\alpha Q$ where $\alpha$ is a positive parameter. Furthermore, as quite common in economic models (see, e.g., Tirole 1988), we impose that price linearly depends on quantity, where the parameter $\gamma$ measures how sensitive price is to quantity. In particular, we use $p(Q, q)=f(Q)-\gamma q$, with $f^{\prime}>0$, i.e. the maximum willingness to pay is larger if the potential stock of customers is large. Typically, when a new product is launched, the stock of potential customers is largest, while at the same time the early adopters are willing to pay the most for the product.

At the optimally determined time $T_{i}$ the firm decides to launch generation $i, i=$ $2, \ldots, N$, of the product. $^{2}$ A major incentive for a new product generation is that with this new generation one can reach more potential consumers again as it might motivate consumers who already bought the old generation to adopt a newer version. When introducing the new product, the demand for old version of the product vanishes, as for instance with cars. The initial stock of potential consumers in each stage is given by the exogenous parameter $Q_{0 i}$, where $i$ denotes the number of the stage, i.e. the generation of the product.

To be able to produce the new product, production capacity needs to be retrofitted. Consequently, at the moment of the new product arrival $T$ production capacity $K$ drops, i.e. $K\left(T_{i}^{+}\right)=\beta K\left(T_{i}^{-}\right)$with $\beta \in[0,1]$, where $T_{i}^{+}$and $T_{i}^{-}$represent the time moment just before and after product arrival, i.e. $K\left(T_{i}^{+}\right)=\lim _{t \rightarrow T_{i}+0} K(t)$.

The costs of introducing a new version of the product are given by the switching costs $S_{i}$. The resulting optimal control model is represented by

$$
\begin{gathered}
\max _{q(\cdot), I(\cdot), T_{i}} \int_{0}^{\infty} e^{-r t}\left((\alpha Q(t)-\gamma q(t)-c) q(t)-I(t)-\frac{\theta}{2} I(t)^{2}\right) d t-\sum_{i=2}^{N} e^{-r T_{i}} S_{i} \\
\dot{Q}(t)=-q(t), \\
\dot{K}(t)=I(t)-\delta K(t), \text { for } t \notin\left\{T_{2}, \ldots, T_{N}\right\},
\end{gathered}
$$

$\overline{2}$ The number of product generations $N$ can either be exogenously given or optimally determined. 


$$
\begin{aligned}
Q\left(T_{i}^{+}\right) & =Q_{0 i} \\
K\left(T_{i}^{+}\right) & =\beta K\left(T_{i}^{-}\right), \quad i=2, \ldots, N . \\
0 & \leq q(t) \leq K(t), \quad I(t) \geq 0, \quad Q(t) \geq 0, \\
Q(0) & =Q_{01}, \quad K(0)=K_{0} .
\end{aligned}
$$

In what follows, in general we omit the time argument $t$ if no confusion is caused.

The model presented here differs to the model analyzed in Seidl et al. (2019) in several ways: here the sales rate $q$ is considered as control variable while in Seidl et al. (2019) it is determined as the minimum of demand and units of the product that can be produced given the capacity. Another big difference is that in Seidl et al. (2019) the price is modeled as state variable which only can change at certain, optimally determined points in time, while here the price follows from the inverse demand function.

\section{Necessary optimality conditions for the $\mathbf{N}$-stage problem}

The Lagrangian is

$$
\begin{aligned}
L= & (\alpha Q-\gamma q-c) q-I-\frac{\theta}{2} I^{2}+\lambda(-q)+\mu(I-\delta K)+v_{1} q \\
& +v_{2}(K-q)+v_{3} I+v_{4} Q
\end{aligned}
$$

where $\lambda$ and $\mu$ are the co-state variables measuring the marginal value of state variables $Q$ and $K$, respectively, see e.g. Grass et al. (2008, p. 109ff). The Lagrange multipliers are denoted by $v_{j}, j=1, \ldots, 4$.

It has to hold that

$$
\begin{aligned}
& L_{q}=\alpha Q-2 \gamma q-c-\lambda+v_{1}-v_{2}=0 \quad \Rightarrow \quad q=\frac{\alpha Q-c-\lambda+\nu_{1}-\nu_{2}}{2 \gamma}, \\
& L_{I}=-1-\theta I+\mu+v_{3}=0 \quad \Rightarrow \quad I=\frac{\mu-1+\nu_{3}}{\theta} .
\end{aligned}
$$

The necessary Legendre Clebsch conditions are fulfilled. The co-state equations are

$$
\begin{aligned}
& \dot{\lambda}=r \lambda-\alpha q-v_{4}, \\
& \dot{\mu}=(r+\delta) \mu-v_{2} .
\end{aligned}
$$

The complementary slackness conditions

$$
v_{1} q=0, \quad v_{2}(K-q)=0, \quad v_{3} I=0, \quad v_{4} Q=0,
$$

must hold. Note that $q=0$ for all $Q \leq \frac{c}{\alpha}$. The reason for this is that if $Q$ is smaller than $\frac{c}{\alpha}$, it is only possible to sell the product making losses. Thus, it is optimal to 
choose the sales rate as zero, meaning the number of potential customers does not decrease any further. Therefore, the non-negativity constraint with respect to $Q$ can never become binding, implying that $v_{4}=0$ for all $t$.

Since $p=\alpha Q-\gamma q$, we have $\dot{p}=\alpha \dot{Q}-\gamma \dot{q}$. Since $\dot{Q}=-q \leq 0$, the price decreases over time (i.e. $\dot{p}<0$ ) if $\dot{q} \geq-\frac{\alpha}{\gamma} q$. We have to distinguish two cases:

1. $q=K$ In this case $\dot{q}=\dot{K}$, consequently we have

$$
\dot{p}=-(\alpha-\gamma \delta) K-\gamma I \text {. }
$$

Due to the non-negativity of the state and control variables, a condition that ensures that the price strictly decreases over time (for $K>0$ ) is $\alpha>\gamma \delta$.

2. $q<K$ In this case we consider $\frac{d L_{q}}{d t}=\alpha \dot{Q}-2 \gamma \dot{q}-\dot{\lambda}=0$, which gives $\dot{q}=-\frac{1}{2 \gamma} r \dot{\lambda}$, which gives in turn

$$
\dot{p}=\frac{1}{2 \gamma}\left((r \gamma+\alpha) \lambda+\alpha c-\alpha^{2} Q\right) .
$$

Here it is not clear whether the price increases or decreases over time.

Concerning capacity investments, it can be seen that

$$
\dot{I}=\frac{1}{\theta} \dot{\mu}, \quad \text { for } I>0
$$

which means that $I$ is non-decreasing if $q<K$.

Due to the discontinuity of the state variables $Q$ and $K$, we use the Impulse Maximum Principle, see Sethi and Thompson (2000, p. 326) and Chahim et al. (2012, Theorem 2.2). In particular, the jumps in the state variables occur at moments that new product versions are introduced, and they are equal to

$$
\begin{aligned}
& Q\left(T_{i}^{+}\right)-Q\left(T_{i}^{-}\right)=Q_{0 i}-Q\left(T_{i}^{-}\right), \\
& K\left(T_{i}^{+}\right)-K\left(T_{i}^{-}\right)=(\beta-1) K\left(T_{i}^{-}\right) .
\end{aligned}
$$

The Impulse Hamiltonian is given by

$$
I H a m=\lambda\left(Q_{0 i}-Q\left(T_{i}^{-}\right)\right)+\mu(\beta-1) K\left(T_{i}^{-}\right) .
$$

When introducing a new product, it has to hold that

$$
\begin{aligned}
\lambda\left(T_{i}^{+}\right)-\lambda\left(T_{i}^{-}\right) & =\lambda\left(T_{i}^{+}\right), \\
\mu\left(T_{i}^{+}\right)-\mu\left(T_{i}^{-}\right) & =\mu\left(T_{i}^{+}\right)(1-\beta),
\end{aligned}
$$

from which we derive that

$$
\lambda\left(T_{i}^{-}\right)=0
$$




$$
\mu\left(T_{i}^{-}\right)=\beta \mu\left(T_{i}^{+}\right) .
$$

Note that the present conditions are reasonable from an interpretation point of view. Namely, the shadow price of the potential consumers has to be zero when a certain generation is abandoned as the firm does not have any utility from them. However, the shadow price of capacity is positive as the firm still can take some advantages of its prior investments into capacity.

Furthermore, it has to hold that

$$
H\left(T_{i}^{-}\right)-H\left(T_{i}^{+}\right)+r S=0 .
$$

\subsection{Steady states}

First of all, we like to mention that there is no steady state with $\hat{K}>0$. This is because $\hat{I}>0$ can only hold if $\hat{\mu}>1$. However, we have $\dot{\mu}=0$ only if $\hat{v}_{2}>0$. This implies that $\hat{q}=\hat{K}$, meaning that $\dot{Q}=0$ only if $\hat{K}=0$. Furthermore, there is no steady state where both controls are in the interior of the admissible control region. However, there exist multiple boundary steady states. There is one steady state with only the constraint $\hat{I} \geq 0$ active, namely,

$$
\begin{aligned}
& \hat{Q}=\frac{c}{\alpha}, \quad \hat{K}=0, \quad \hat{\lambda}=0, \quad \hat{\mu}=0, \quad \hat{q}=0, \quad \hat{I}=0, \quad \hat{v}_{1}=0, \quad \hat{v}_{2}=0, \\
& \hat{v}_{3}=1, \quad \hat{v}_{4}=0 .
\end{aligned}
$$

The Jacobian evaluated at the steady state is

$$
J=\left(\begin{array}{cccc}
-\frac{\alpha}{2 \gamma} & 0 & \frac{1}{2 \gamma} & 0 \\
0 & -\delta & 0 & -1 \\
-\frac{\alpha^{2}}{2 \gamma} & 0 & r+\frac{\alpha}{2 \gamma} & 0 \\
0 & 0 & 0 & r+\delta
\end{array}\right) .
$$

Its eigenvalues are $\xi_{1}=-\delta, \xi_{2}=r+\delta, \xi_{3,4}=\frac{1}{2 \gamma}(r \gamma \pm \sqrt{r \gamma(r \gamma+2 \alpha)})$, which implies that two of the eigenvalues are always positive and two are negative.

It can be seen that the steady state level of potential consumers increases in the production costs $c$ and decreases in $\alpha$ which measures the impact of the consumer stock on the price. Note, however, that at the steady state itself, there are no sales. This is inherent to the model as sales decrease the stock of potential customers and $\dot{Q}$ cannot be zero if $\hat{q}>0$. The particular steady state level $\hat{Q}$ is such that the price equals production costs, below that level potential consumers could only be motivated to buy the product if its price is below costs which is not profitable for the firm.

At the steady state $\hat{v}_{2}=0$, i.e. the constraint $K \geq q$ is not active at the steady state. Since the steady state value $\hat{\mu}$ is zero and (2) is strictly positive for any $\mu(t)>0$ when $v_{2}(t)=0$ this means that the steady state can only be approached if $v_{2}(t)>0$ for some $t \geq 0$ or if $\mu(t)=0$ for $t \in[0, \infty)$. The second case would imply that it is never profitable for a firm to invest into capacity, which would be the case if investment costs 
are too high. Note that in this case there could only be a feasible solution path with $Q_{0}>\hat{Q}$ approaching the steady state if $K_{0}>0$ as $q>0$ only if $K>0$. Obviously, the more interesting case is when $\nu_{2}(t)>0$ for some $t \geq 0$. Since $I>0$ if $\mu>1$ investments into capacities are zero in vicinity of the steady state which makes sense as there are no sales at the steady state. Furthermore, it only makes sense to invest into capacities when $q=K$ when approaching the steady state as there is no incentive to overinvest into capacities.

There is another steady state where $q \leq K$ is active, i.e.,

$$
\begin{aligned}
& \hat{Q}=\frac{r+\delta+c}{\alpha}, \hat{K}=0, \quad \hat{\lambda}=0, \quad \hat{\mu}=0, \quad \hat{q}=0, \hat{I}=0, \quad \hat{v}_{1}=0, \quad \hat{v}_{2}=r+\delta, \\
& \hat{v}_{3}=0, \quad \hat{v}_{4}=0
\end{aligned}
$$

with the Jacobian

$$
J=\left(\begin{array}{cccc}
0 & -1 & 0 & 0 \\
0 & -\delta & 0 & \frac{1}{\theta} \\
0 & -\alpha & r & 0 \\
-\alpha & 2 \gamma & 1 & r+\delta
\end{array}\right)
$$

and its eigenvalues

$$
\begin{aligned}
\chi_{1,2,3,4}= & \frac{1}{2}\left(r \pm \theta\left[\theta(r+\delta)^{2}+4 \gamma+\theta \delta \pm\left((2 \gamma+\theta r \delta)^{2}\right.\right.\right. \\
& \left.\left.\left.+\theta\left(2 \theta \delta^{3} r+4 \gamma \delta^{2}+\theta \delta^{4}-4 \alpha r\right)\right)^{0.5}\right]\right)^{0.5} .
\end{aligned}
$$

Note that these eigenvalues can be complex. Furthermore, since

$$
q=0 \quad \forall Q \leq \frac{c}{\alpha},
$$

(below which the price, which is $p=\alpha Q-\gamma q$, is too small to compensate production $\operatorname{costs} c$ ) there is a manifold of steady states with

$$
\begin{aligned}
& \hat{Q} \in\left[0, \frac{c}{\alpha}\right], \\
& \hat{K}=0, \quad \hat{\lambda}=0, \quad \hat{\mu}=0, \quad \hat{q}=0, \quad \hat{I}=0, \quad \hat{v}_{1}=-(\alpha \hat{Q}-c), \quad \hat{v}_{2}=0 \\
& \hat{v}_{3}=1, \quad \hat{v}_{4}=0 .
\end{aligned}
$$

\subsection{Single-stage problem}

To gain a better understanding of the problem let us first consider the number of stages, $N$, to be fixed. In the most simple case, there is just one stage, meaning that there is 

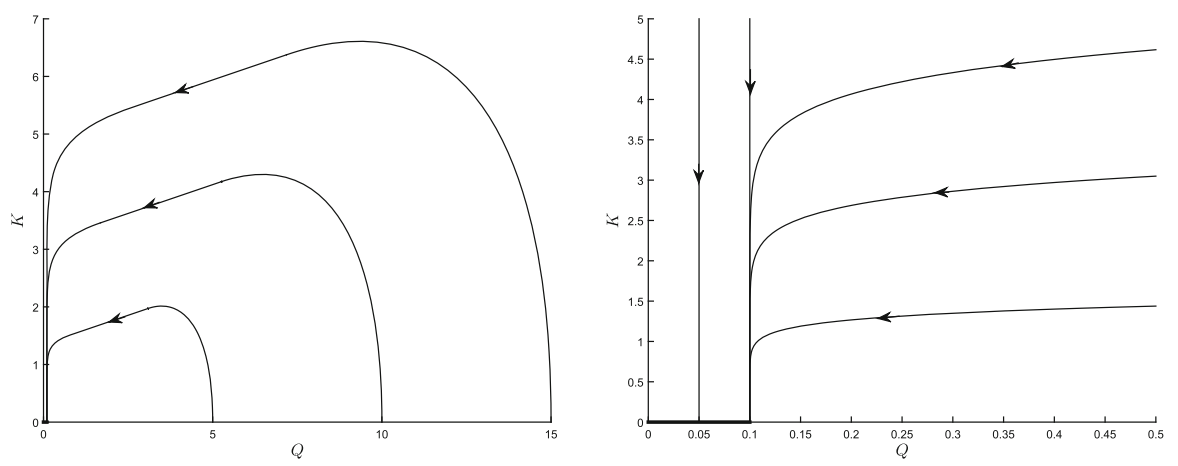

Fig. 1 Phase portrait-single-stage problem

just one product which is never replaced by a newer version. As parameter values we used

$$
r=0.1, \quad \alpha=10, \quad c=1, \quad \beta=0.5, \quad \gamma=0.1, \quad \delta=0.2, \quad \theta=1
$$

The parameter values above are arbitrarily chosen in the sense that they are not empirically validated. The present paper, however uses the same parameter values as in Seidl et al. (2019), which allows us to compare the solutions. We can find the steady states described above, however, numerical calculations comparing solutions leading to the different steady states indicate that if $Q_{0 N} \geq \frac{c}{\alpha}$ approaching the steady state (3), i.e.

$$
\begin{aligned}
& \hat{Q}=\frac{c}{\alpha}=0.1, \quad \hat{K}=0, \quad \hat{\lambda}=0, \quad \hat{\mu}=0, \quad \hat{q}=0, \quad \hat{I}=0, \quad \hat{v}_{1}=0, \\
& \hat{v}_{2}=0, \quad \hat{v}_{3}=1, \quad \hat{v}_{4}=0
\end{aligned}
$$

is optimal. For $Q_{0 N}<\frac{c}{\alpha}$ it is optimal to have the sales rate and investments equal to zero in the terminal stage, implying that one approaches the manifold of steady states (4). In the subsequent, we only consider values of $Q_{0 i}$ greater than $\frac{c}{\alpha}$ for all $i=1, \ldots, N$.

The phase portrait is shown in Fig. 1, where the right panel is a zooming of the left panel. If $Q_{01}<0.1$, selling the products would only be possible making losses, thus it is optimal then to produce nothing, invest nothing into production capacities, and let the capacities just depreciate until it becomes zero.

If, however, $Q_{01}>0.1$, then it can pay off to invest into capacity. Figure 2 shows a time path of a solution starting at $Q(0)=10, K(0)=0$. Initially it is optimal to invest much into capacity so that it can increase. The quantity of the good sold to the consumers equals the production capacity, i.e. the firm produces as much as possible. The stock of potential consumers decreases, thus one can also decrease investments into the capacity. After some time investments will become zero and due to the smaller number of potential consumers, and less will be produced/sold than possible, i.e. there is actually more capacity available than necessary. A firm is interested to sell its 

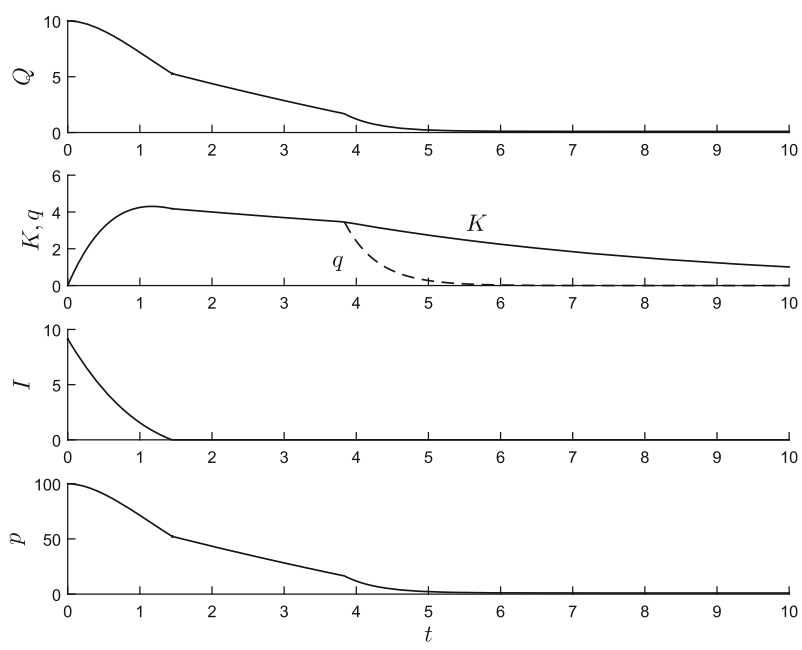

Fig. 2 Time path for $Q(0)=10, K(0)=0$ - single-stage problem

product initially fast so it overinvests into capacity in the sense that it does not operate with full capacity for the entire planning horizon, particularly not when the market for the product has grown small. It is reasonable that when there are many potential consumers the price is high, and low when there are only few of them, and equals production costs in the long run.

Since a non-constant price allows to adapt the price to the heterogeneous amount that consumers are willing to pay for the durable product, it is not surprising that sales are larger in the present model than in Seidl et al. (2019). Consequently, investments are also larger here. The time for which sales equal capacity is about twice as long as for a constant price, which also relates to the higher sales in the present paper.

\subsection{Two-generation problem}

The next step is to analyze what happens if there is a second stage, i.e. the first product is replaced after an optimally determined time $T_{2}$ by the second generation of the product. Then the number of potential consumers goes up, i.e. $Q\left(T_{2}^{+}\right)=Q_{02}$ and the capacity goes down, i.e. $K\left(T_{2}^{+}\right)=\beta K\left(T^{-}\right)$.

Figure 3 shows an illustrative solution path starting at $Q(0)=10, K(0)=0$. The parameters are the same for both product generations. During the first stage, when generation one is produced and sold, one always sells as much as one can produce. This also holds true for the beginning of the second stage, but only for some time. Investments into capacity are higher in the beginning, but are decreasing. When introducing the second generation of the product, one increases investments again to compensate the loss of capacity due to retrofitting. Note that investments are not as high in the second stage as in the first stage, which makes sense as one only has a utility from investments as long as the market for the product is sufficiently large. The investments during the first stage have a positive impact on the capacity for the second 

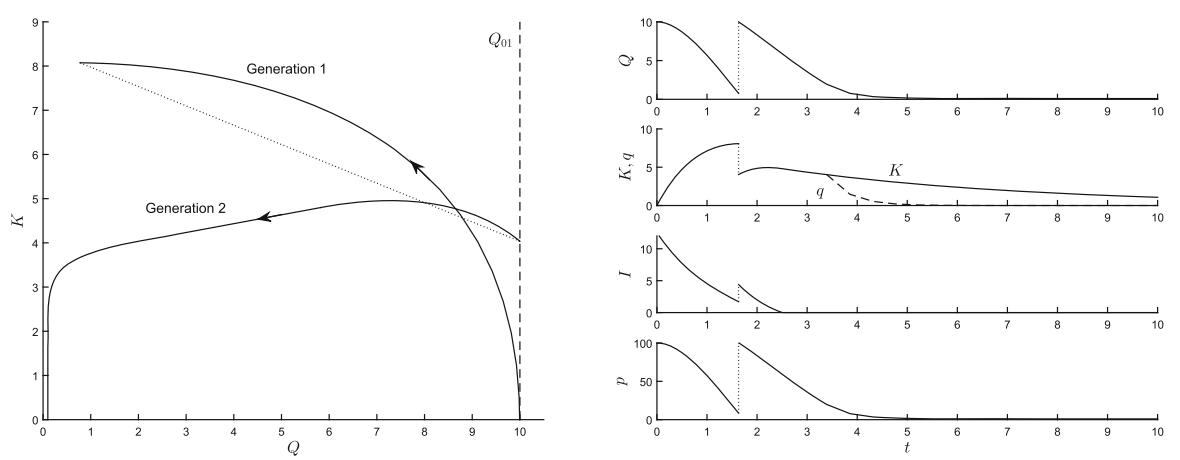

Fig. 3 Two-stage problem, phase portrait and time path

generation as not all of it is lost when switching to the new version of the product. As no further generation is introduced, the market gets saturated after some time.

Note that the intersection of the solution path with itself in the phase space is not (only) because of the discontinuity of the state variables at the switching time. Later on we will see that this phenomenon does not occur when the number of switches is not restricted. This makes sense as [following the proof of the monotonicity theorem presented in Hartl (1987)] without restriction on the number of switches it cannot be optimal to pursue two different strategies w.r.t the continuous control instruments at the same point in the state space if the problem is autonomous.

Comparing the outcome to Seidl et al. (2019), it can be seen that the non-constant price leads to a higher switching time. During an initial generation of the product a firm will choose a low sales rate implying a high price. In this manner it can compensate the investments and keep initial demand requiring capacity low. If the price remains constant for the entire period in which a generation of the product is sold, this means that there are more consumers who are not able to afford or willing to purchase the product. Thus, by adapting the price one can gain more consumers. Of course this requires also more capacity and investments. Note, however, profits are higher for the case of a non-constant price.

Figure 4 shows what happens if the market for the second generation of the product is much smaller than for the first generation. The different sizes of the potential buyers of each generation can relate to differences in the functionality and/or the quality of the product. In this case it does not pay as much as before to invest into capacity, because less of it is required to meet demand in the second stage. Unlike for larger $Q_{02}$, the firm stops investing into capacity already during production of the first generation, as can be seen in Fig. 4. Consequently, the overal investments into capacity are less than before. Note, however, that there is also less unused capacity in the second stage. Furthermore, the price increase after the introduction of the new generation is not as high as before due to the smaller market size.

The impact of the market potential for the second version of the product is summarized in the left panel of Fig. 5. There one can see that the switching time $T_{2}$ is higher if $Q_{02}$ is low. The reason is that a firm wants to reach more potential consumers before switching, because there are less to be reached after switching than 

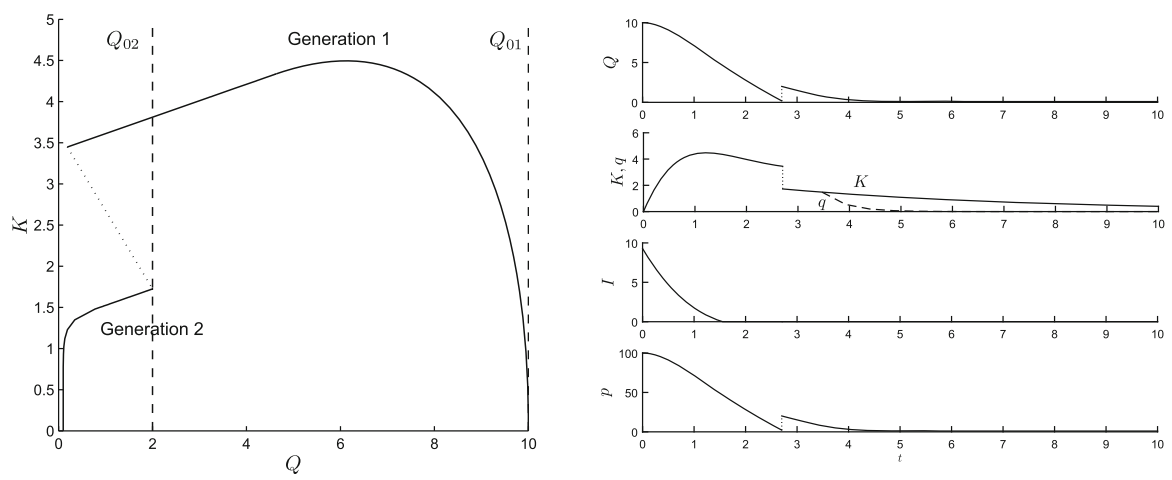

Fig. 4 Two-stage problem, phase portrait and time path for $Q_{02}=2$
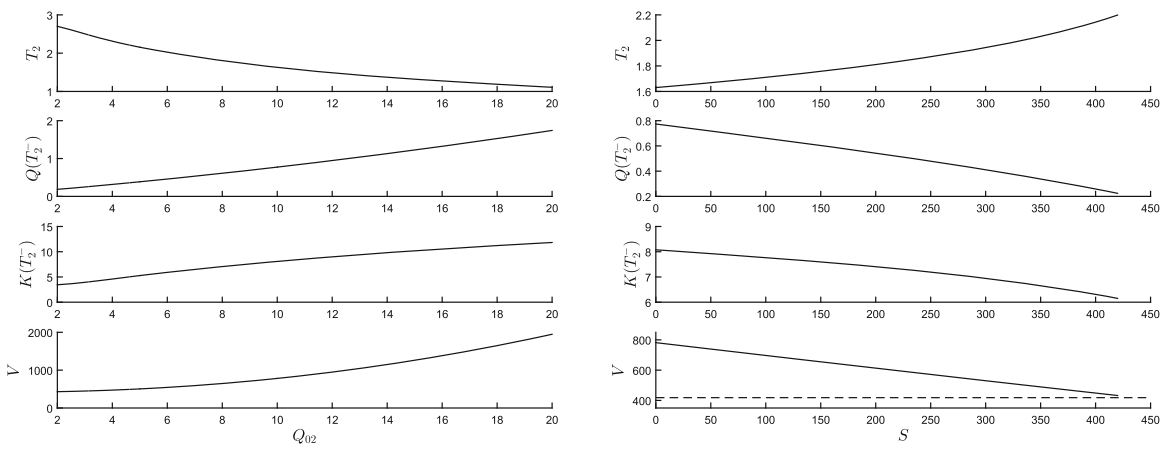

Fig. 5 Sensitivity analysis w.r.t. the number of potential customers in stage $2, Q_{02}$, and w.r.t. the switching costs $S$ for $Q(0)=10$ and $K(0)=0$

before. Consequently, the market size before switching, $Q\left(T_{2}^{-}\right)$, is lower if $Q_{02}$ is low. More investments into capacity are required to satisfy demand after switching if $Q_{02}$ is high. Therefore, it makes sense then that capacity before switching is higher. Not surprisingly, profits are higher if the market is also large in the second stage.

The impact of switching costs $S$ is shown in the right panel of Fig. 5. An increase of the switching costs leads to an increase of the switching time $T_{2}$ and a decrease of the market size before switching $Q\left(T_{2}^{-}\right)$. Same as before, one would try to exploit the availability of potential consumers in the first stage, accepting a delay of the high initial profits of the second stage. When switching costs are high, a firm is less able to invest into capacity, therefore the level of capacity before switching is lower if switching costs are high. When the switching costs are too high, it does not pay off to switch at all and it is never optimal to introduce the new version of the product.

The impact of parameter $\beta$, which describes the extent to which one can reuse the capacity for the production of the first product version for the second, is shown in Fig. 6. Interestingly, the switching time $T_{2}$ depends non-monotonically on parameter $\beta$. For low $\beta$, capacity is increasing in $\beta$ : this is a value effect, because it is more valuable to invest in capacity if more of the capacity can also be used after the switch. For $\beta$ high, capacity is decreasing in $\beta$ : this is an expansion effect, because if more 

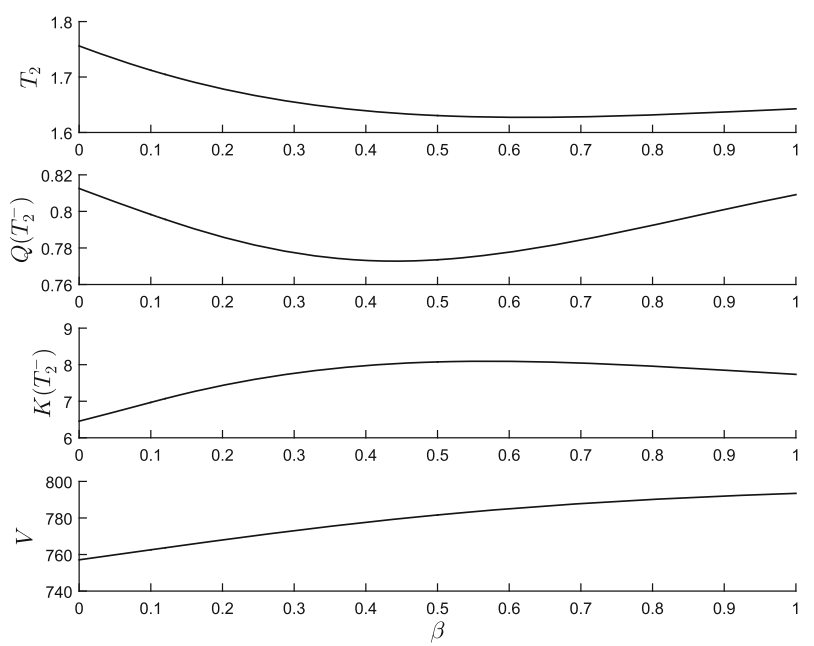

Fig. 6 Sensitivity analysis w.r.t. $\beta$, i.e. the extent to which capacity can be reused in stage 2 for $Q(0)=10$ and $K(0)=0$

of the capacity can be used after the switch then there is no need to build up capacity so much before the switch. $T_{2}$ behaves in the opposite way than capacity: for small $\beta$ switching time $T_{2}$ decreases with $\beta$ because it becomes more attractive to switch if you can use more of your old capacity after the switch. For $\beta$ large, $T_{2}$ increases with $\beta$ because the firm has invested less all along implying that the upper bound for sales is lower. Thus, the stock of potential consumers stays higher and the market is profitable for a longer period of time.

Note that this non-monotonicity is also observed in the problem where the price is assumed to be constant, see Seidl et al. (2019). Comparing the solutions, it can be seen that the switching time is larger for a non-constant price. The number of potential consumers before switching to the next generation is significantly smaller. This is not surprising as the non-constant price allows to charge a high price first to compensate for capacity investments, and then to lower it to allow a larger number of consumers to purchase the product. The capacity before introducing a new product generation is larger for the non-constant price, because one can afford to invest more.

If the capacity cannot be reused for the second stage (i.e. $\beta=0$ ), it makes sense to exploit available capacity to a larger extent already in the first stage. Figure 7 shows the solution path starting at $Q(0)=10, K(0)=0$ in the state space and the corresponding time paths. Compared to the base case, where $\beta=0.5$, the main difference is the investment strategy: Since one cannot reuse the capacity, it does not make sense to invest in it at all during the time before introducing the new version, thus, $I\left(T_{2}^{-}\right)=0$. However, after the new generation of the product is introduced higher investments into capacity are required as one needs to build up capacity again from zero.

When one can completely reuse the capacities $(\beta=1)$ built up for the first generation of the product, the switching time $T_{2}$ is higher than in the base case for $\beta=0.5$. In this case investments are more efficient as less capacity gets lost when switching and 

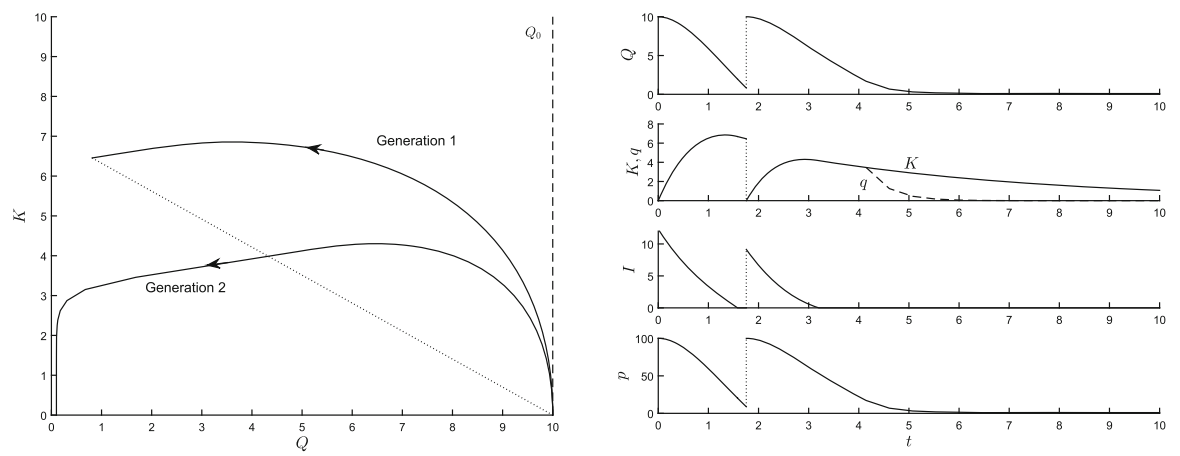

Fig. 7 Two-stage problem, phase portrait and time path
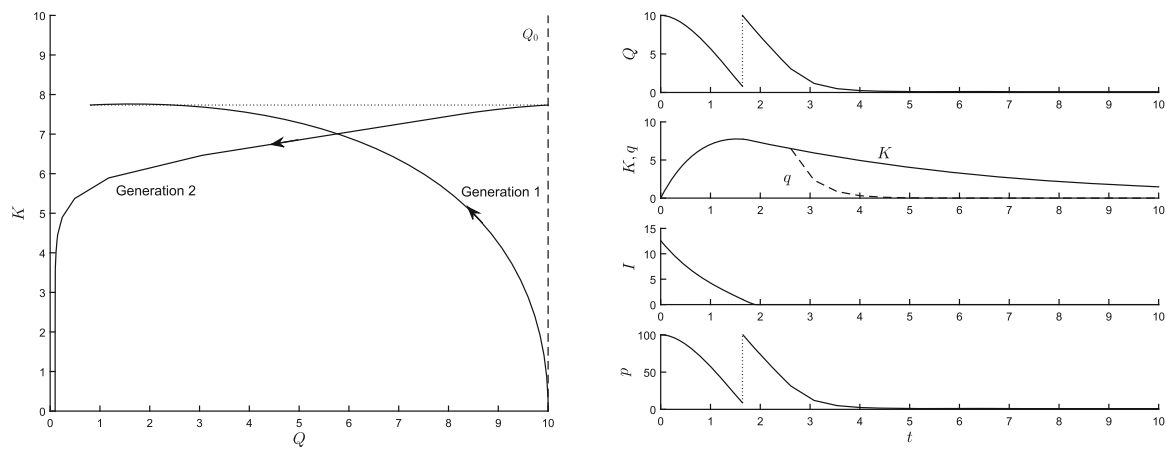

Fig. 8 Two-stage problem, phase portrait and time path

therefore a firm can afford to invest less, i.e. for the intermediate case it makes sense to invest more to compensate for the capacity loss in order to be able to continue high sales after switching. Unlike the case of very low $\beta$ these investments are not lost, and unlike the case of high $\beta$ the investments are needed to have sufficient capacity in the beginning of the second stage. Thus, for $\beta=1$ one can afford to exploit the available number of potential consumers (who generate less profits as the price is low before switching) without worrying too much about the depreciation of capacity when less consumers are available and thus less capacity is required, see Fig. 8.

\subsection{Multi-generation problem}

In the following section we consider what happens if we increase the (fixed) number of stages. The case with three generations of the product is shown in Fig. 9. Again, the initial state value is $Q(0)=10$ and $K(0)=0$. The highest investments into capacity are made in the first stage. Of course, this is necessary to be able to sell anything in the beginning when there is no capacity available otherwise. However, despite the downfall of capacity when switching to the new version of the product, one profits from these investment in the subsequent stages most. After switching to the second stage, one increases investments into capacity to compensate for the loss due 

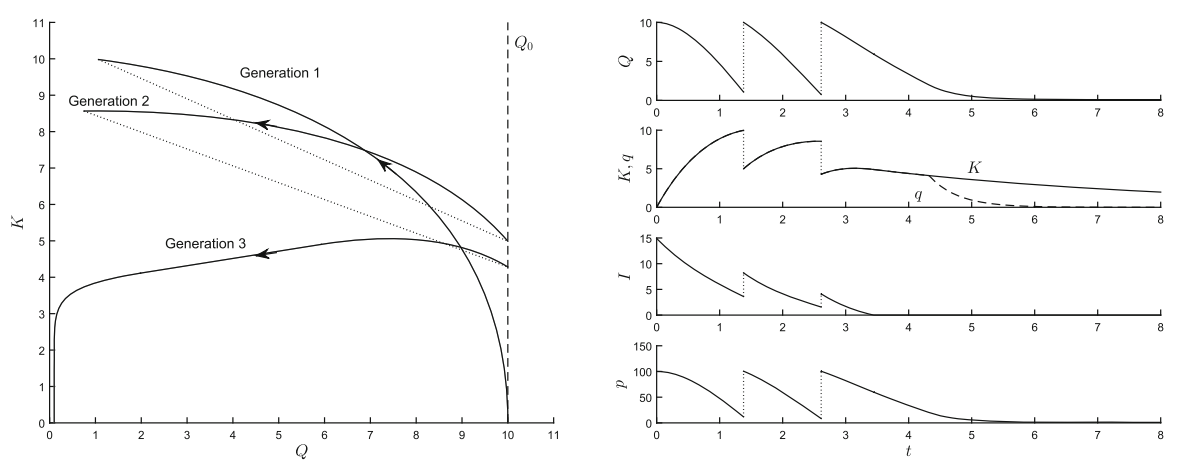

Fig. 9 Three-stage problem
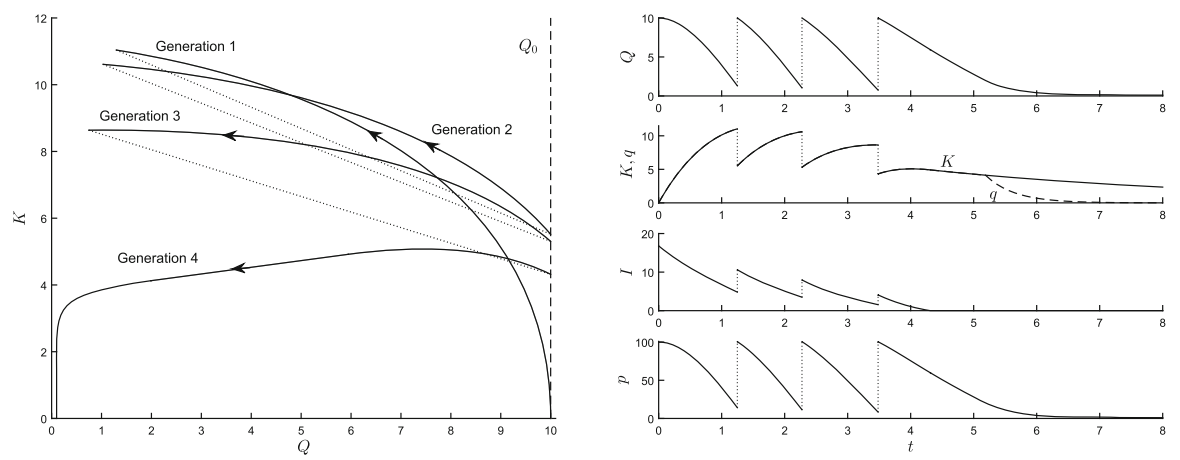

Fig. 10 Four-stage problem

to switching. However, as one does not lose capacity in the final, third stage anymore, less investments are needed.

What happens if we increase the number of generations to four can be seen in Fig. 10. The picture is similar to the three stage case: in the first stage one invests most, then investments in each subsequent stages are less as the firm does not need to compensate the capacity loss due to the subsequent introduction of new product generations as much.

The case with 100 product generations can be seen in Fig. 11. The idea behind choosing such a high number of stages is not only to analyze how such a large number of switches affects the investment and pricing strategy per se but also to check whether the profits of the firm increase or decrease in the number of switches (note that the conditions for switching from one stage to the next are only necessary conditions). If it increases, it is an indication that for an actual unrestricted number of switches a periodic solution with infinite switches is optimal. One can see in Fig. 11 that the solution path for 100 generations of the product approaches the periodic solution (for details on the periodic solution, see the next section of the paper). Figure 12 shows the dependency of the value of the objective on the number of generations $N$. It can be seen that the value of the objective approaches a fixed level which is the actual value of the objective for a periodic solution with an infinite number of product generations. 

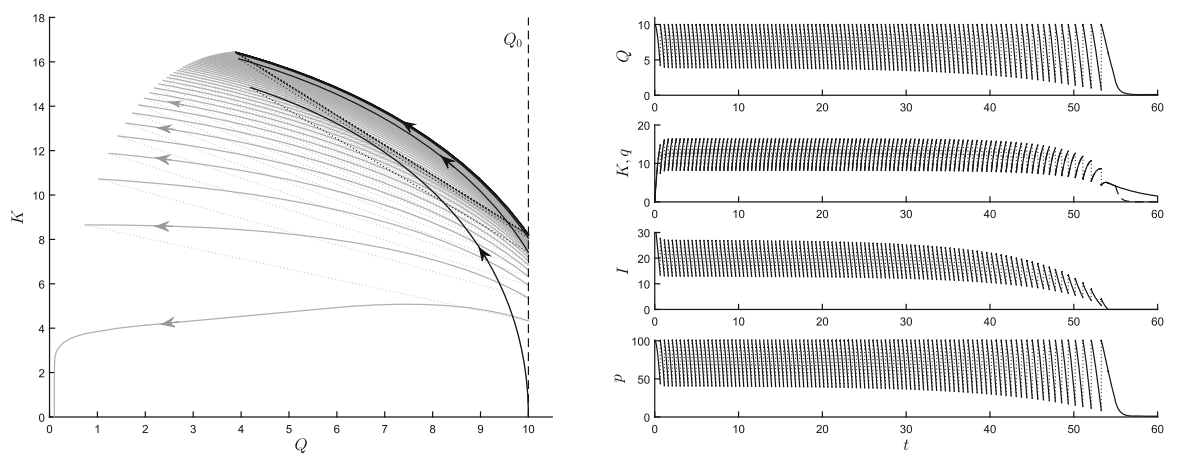

Fig. 11 100-stage problem

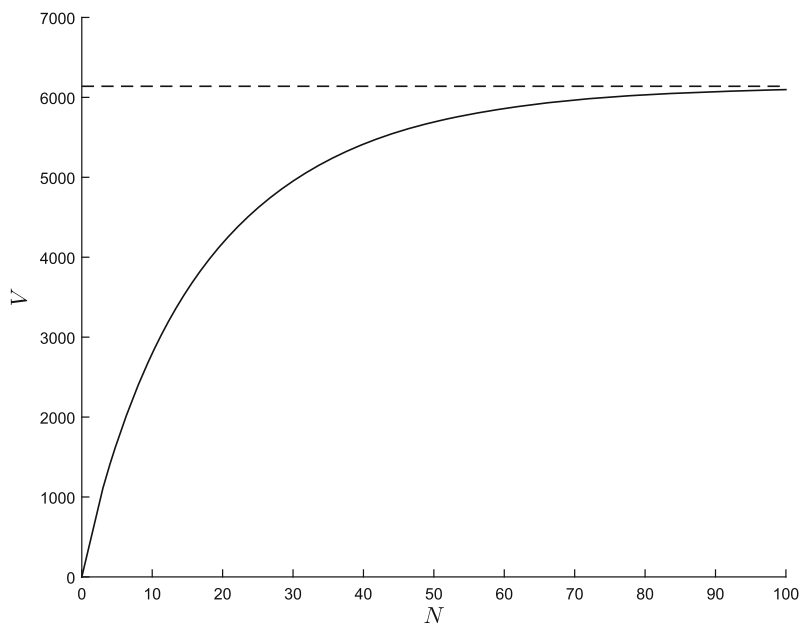

Fig. 12 Value of the objective function depending on the number of switches for $Q(0)=10$ and $K(0)=0$. The dashed line depicts for comparison the objective value for $N=\infty$ (periodic solution)

Concerning the optimal strategy for such a high number of stages, it is optimal at the beginning of the planning horizon to have high investments such that the capacity increases and the losses of capacity due to switching are compensated. For the initial generations, the investments are so large that the capacity after the introduction of a new generation exceeds the capacity after the launch of the preceding one. However, after some time, when there are not many further switches possible, the effects of the market saturation play a larger and larger role again and investment get smaller and smaller.

\subsection{Periodic solution: infinite generations}

To find the periodic solution numerically, we formulate the following boundary conditions, see also Seidl (2012). The optimally determined period of the cycle is denoted by $\bar{T}$. Since the problem is autonomous, we consider the solution on the interval 

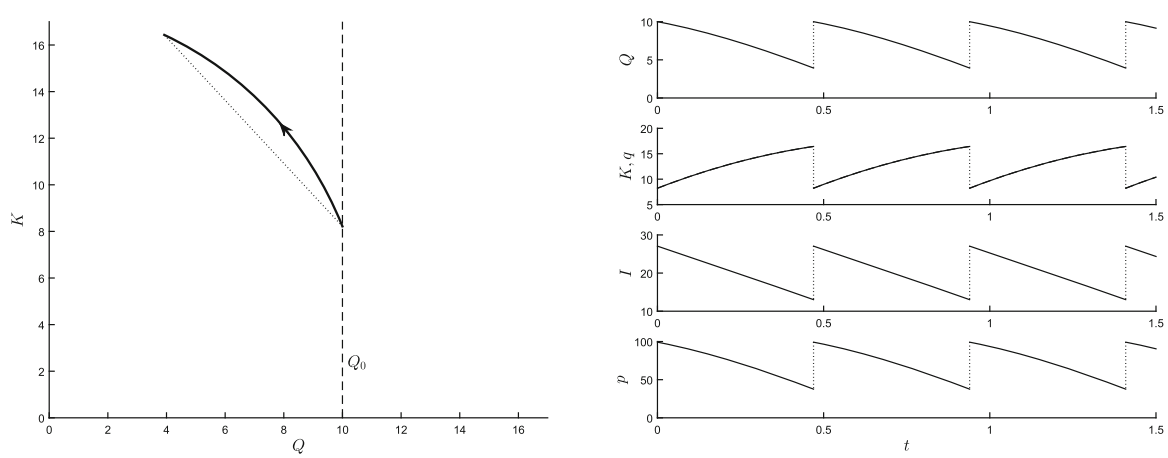

Fig. 13 Periodic solution in the phase space (left panel) and corresponding time path (right panel)

$t \in[0, \bar{T}]$. It has to hold that

$$
\begin{aligned}
Q(0) & =Q_{0} \\
K(0) & =\beta K(\bar{T}) \\
\lambda(\bar{T}) & =0 \\
\mu(\bar{T}) & =\beta \mu(0) \\
H(\bar{T}) & =H(0)+r S
\end{aligned}
$$

Since $\dot{\mu}>0$ for any $q<K$ [see (2)], this means that a periodic solution with $q$ being in the interior of the admissible control region for all $t \in[0, \bar{T}]$ is only possible if $\beta$ is greater such that condition (8) can be fulfilled (for positive values of the co-state). Note that then, even though a periodic solution might occur, it does not necessarily has to be optimal. Note that a negative value of $\mu(t)$ implies $I(t)=0$. Since $\beta>1$ is not particularly meaningful, we focus on the case where the control constraint $q \leq K$ becomes active for some time $t \in\left[\tau_{1}, \tau_{2}\right]$, where $\tau_{1} \geq 0$ and $\tau_{2} \leq \bar{T}$.

Consequently, at least at some part of the periodic solution $q=K$. Considering this constraint in more detail we find that Lagrange multiplier $\nu_{2} \geq 0$ only if

$$
\alpha Q-2 \gamma K-c-\lambda \geq 0
$$

The canonical system can be written as

$$
\begin{aligned}
\dot{Q} & =-K, \\
\dot{K} & =\frac{\mu-1+v_{3}}{\theta}-\delta K, \\
\dot{\lambda} & =r \lambda-\alpha K-v_{4}, \\
\dot{\mu} & =(r+\delta) \mu-v_{2}=(r+\delta) \mu-(\alpha Q-2 \gamma K-c-\lambda) .
\end{aligned}
$$

Figure 13 shows a solution for the present parameter values which fulfills the boundary conditions (5)-(9). On the whole periodic solution it holds that $q=K$, i.e. sales equal capacity. Investments into capacity are the highest at the beginning of 

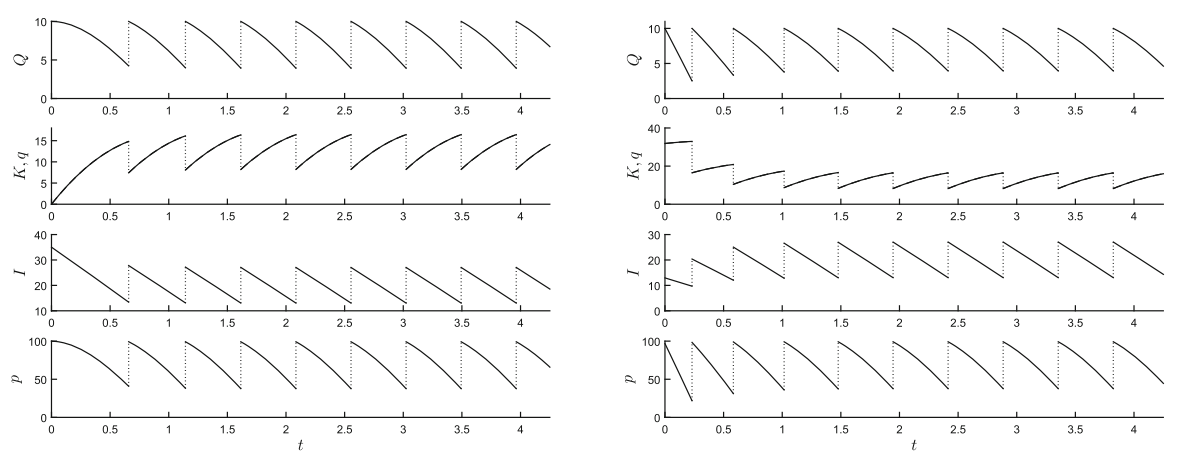

Fig. 14 Time paths approaching the cycle with a low initial capacity (left panel) and a high initial capacity (right panel)

each product release, but decrease over time. Note, however, these investments are still large enough so that $K$ increases over time except at the switching point. It makes sense to invest most when introducing the new product, first to compensate for the capacity loss due to switching and second because the market is the largest then.

The period of the cycle is slightly larger than for the periodic solution obtained in Seidl et al. (2019) where optimal price is kept constant for each product generation. Note, however, that the investments into capacity are much larger, leading to faster sales and a faster decline of the number of potential consumers. One can afford this for the non-constant price, because here one can exploit the fact that some consumers who are willing to pay more for the same product as others by adapting the price. Thus, the non-constant price implies that one can charge a high price first and then lower it so that there are more consumers who can actually afford the product.

The time path for solution paths approaching the cycle can be seen in Fig. 14, the corresponding phase portrait is shown in Fig. 15. If the capacity is zero in the beginning one has to invest much to be able to produce and sell the product. Thus, capacity and sales increase. After some time, there are so little potential consumers left so that it is optimal to switch to the next stage - then the firm is also able to charge a higher price. In the second stage it is not optimal/necessary to invest as much as before, capacity still increases over time except at the switching time. Note that the switching time is the highest in the first stage. This is because one cannot sell as much in the beginning as one does not have the means to produce as much as necessary and therefore one needs longer to capture the potential consumers.

When starting with a high initial capacity, a firm does not need to invest as much as before. Still, the investment strategy is to invest so much that the capacity still increases over time (at least when the capacity is not too high) to compensate for the capacity losses due to the introduction of the new product generation. The capacity decreases by assumption at the switching points. After switching it is therefore necessary to invest more. Note, however, the investment level is below that of the periodic solution at least in the first couple of stages.

The impact of the switching costs $S$ on the periodic solution is investigated in Fig. 16. The objective value of starting directly on the periodic solution, i.e. on $Q(0)=10$ and 


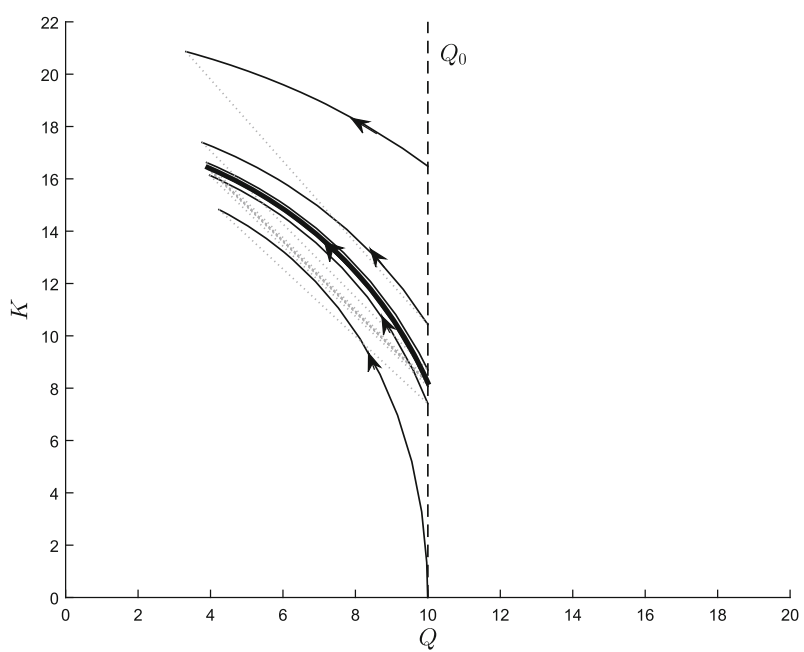

Fig. 15 Phase portrait where the number of switches is not restricted

$K(0)=K^{+}$, where $K^{+}=K\left(\bar{T}^{+}\right)$, is depicted by the solid line (i). Not surprisingly the value of the objective of the periodic solution decreases in $S$. Note, however, that $K\left(\bar{T}^{+}\right)$differs for different values of $S$, in particular it decreases in $S$. For comparison, the value of the objective is shown if (ii) just one switch is allowed and (iii) no switch is allowed. One can see that for small switching costs, the objective value is always above that for solutions with a restricted number of switches. If the switching costs become too high, then it becomes optimal to never switch at all. The switching time increases with switching costs. This has presumably two reasons: first one cannot afford to invest as much into capacity as before and second one needs to exploit the customer base of each stage as much as possible.

Note that when the switching costs are high, something interesting happens: Fig. 17 illustrates that for high switching costs, the control constraint $q \leq K$ can become inactive, i.e. initially it is optimal to sell as much as possible, but closely before switching to the next stage this is not the case anymore. Furthermore, Fig. 17 shows that if switching costs are higher the firm invests less in such a project, it does not pay off. The increase of the investments over time at the end of the first stage imply that then one already builds up capacity for the next stage, so that one can start with high sales then. The strategy has the practical reason that one needs to exploit the available potential customers as far as possible and when the number of potential customers is low, then the inverse demand function suggests that sales should be kept low to be able to charge a positive price.

The left panel of Fig. 18 shows the impact of parameter $\beta$, i.e. the extent to which one can reuse capacity in the next stage on the periodic solution. Obviously, the value of the objective increases when less adjustments need to be made for the production of the new generation of the product. Because of a higher $\beta$, less investments are needed to keep up a high capacity and one can afford to not capture less potential customers. 

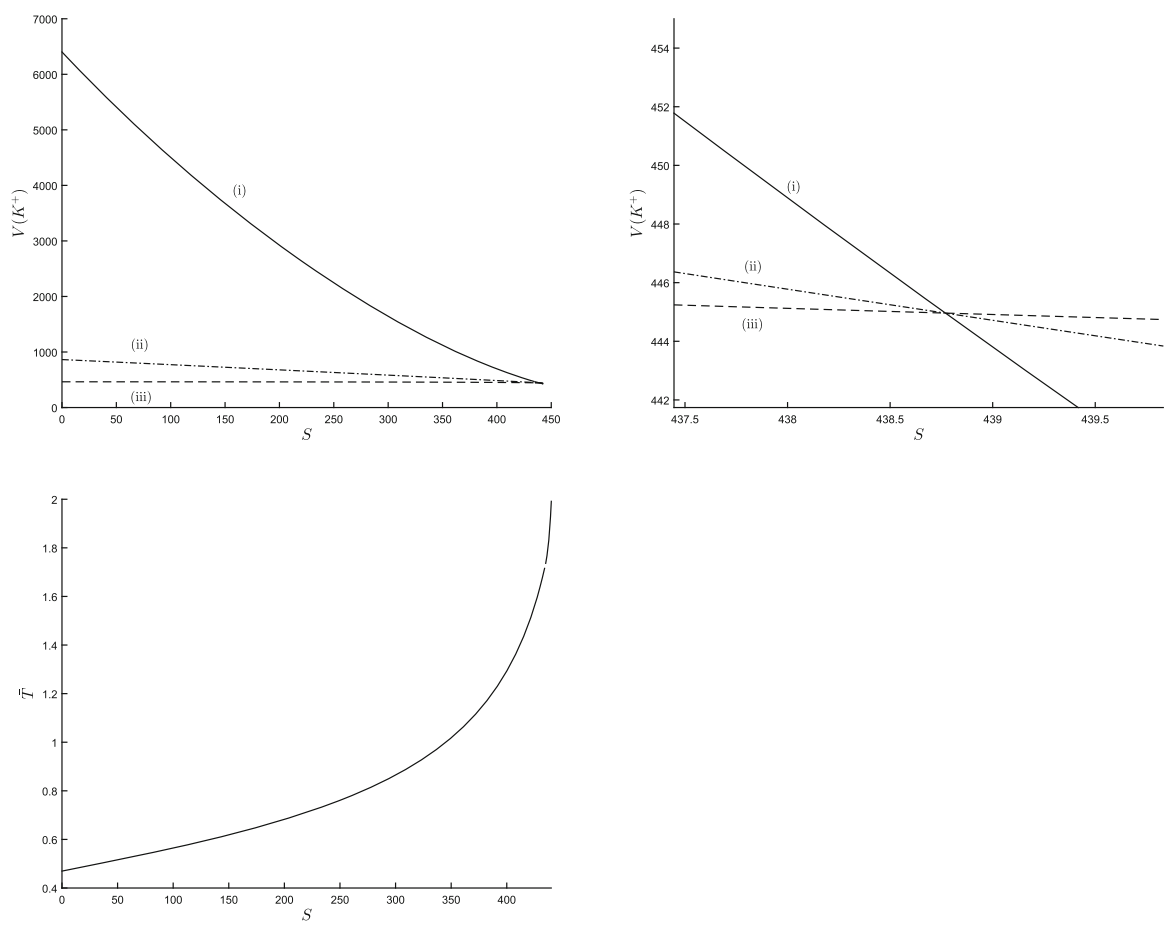

Fig. 16 Impact of switching costs on the periodic solution (i) with respect to the value of the objective (upper panel) and with respect to the switching time (lower panel)
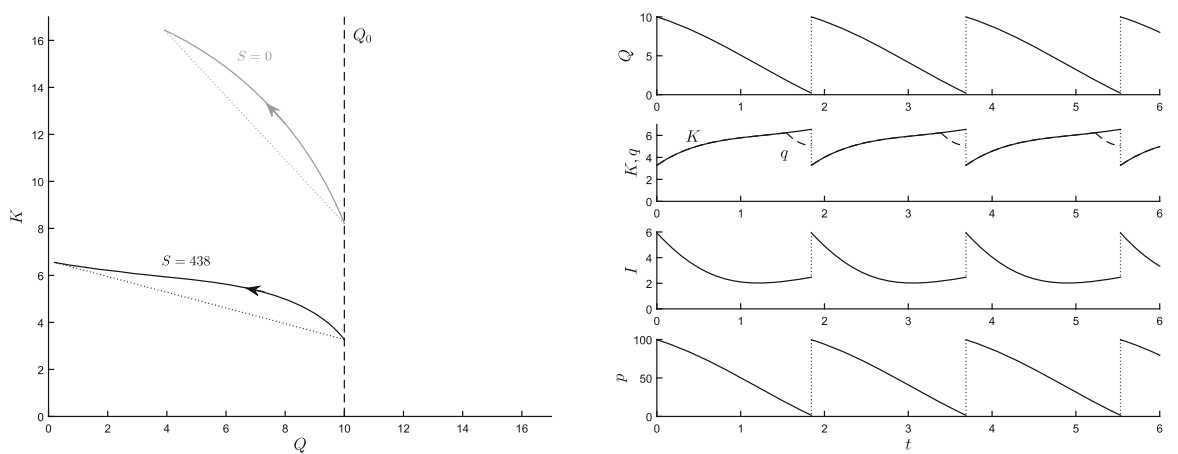

Fig. 17 Phase portrait and time path of a periodic solution for high switching costs

The right panel of Fig. 18 shows the impact of the market size on the decision to introduce a new generation of the product. What is interesting is that the switching time decreases in $Q_{0}$. If the market is large then one has an incentive to switch sooner as there are more people who would buy the product. Note, however, that the difference between the initial and the final state value on the periodic solution decreases when $Q_{0}$ becomes smaller. 

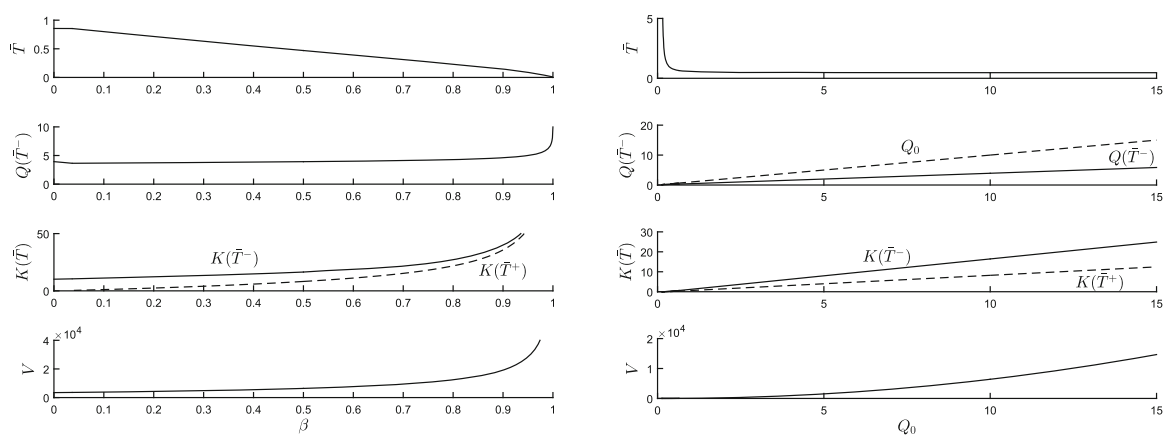

Fig. 18 Impact of the extent at which one can reuse capacity in the next stage and on the initial number of potential customers in each stage on the periodic solution
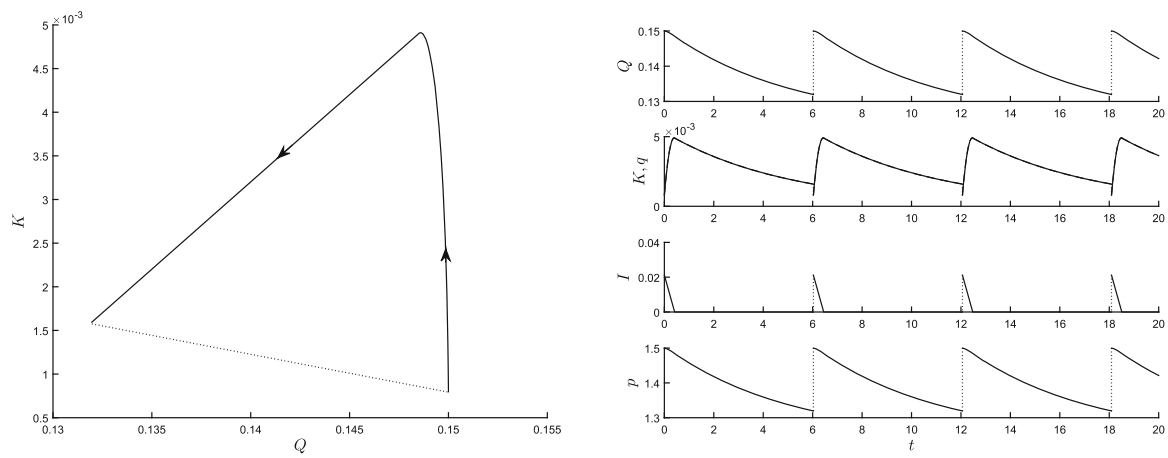

Fig. 19 Periodic solution for $Q_{0}=0.15$

We considered the periodic solution for for an intermediate and a low market potential. Figure 19 illustrates the periodic solution for $Q_{0}=0.15$ in the phase space in the left panel and the time path in the right panel. One can observe that investing into capacity becomes zero for some time before switching leading to a decrease of capacity. Obviously when there are only a few potential customers investment do not pay off so much. Thus, sales rates are lower than in the base case and the number of potential customers decreases more slowly. One can conclude that the lower $Q_{0}$ gets the less investments pay off.

The impact of other parameters was also investigated: The effect of a change of parameter $c$, the production costs, is not particularly large: When $c$ increases the period of the cycle slightly increases because the firm invests less. Thus, the upper bound on sales is lower which makes that the firm takes a longer time to serve the demand of the stock of potential consumers. The customer base before switching also increases slightly, and the capacity at $\bar{T}^{-}$becomes smaller, thus one would produce and sell less. Obviously, profits decrease in $c$. An increase of the depreciation rate $\delta$ has a similar effect, while an increase of parameter $\alpha$, which relates the number of potential consumers to the maximum possible sales, has the opposite effect. 


\section{Conclusion}

The durable goods model we considered led to the following main results. First, investment in capacity is largest directly after the launch of the new product. The reason is twofold. Capacity needs to be built up, and the stock of potential consumers is the largest at the beginning of a period in which a certain product is put up for sale. Second, we found that the switching time is non-monotonic in $\beta$, which is the part of the capacity that can be rolled over to the production process of the new good. However, in the periodic solution it holds that the firm will switch sooner for a larger value of $\beta$. Third, firms produce up to capacity when switching costs are not too high. Fourth, switching costs reduce investments considerably. The reason is that switching is less attractive. Therefore the firm keeps on selling the same product for a longer time so that not much capacity is needed to serve all consumers who want to buy.

Potential extensions for the the present model would be to consider the consumers' behavior in more detail. For example, one could analyze how the optimal strategy is affected if consumers are impatient, i.e. a certain fraction of the consumers which is not able to purchase the product due to the limited capacity loses interest. Furthermore, one could study the impact of network effects. Then there would be an inflow to the number of potential consumers related to the previous sales of the product.

Acknowledgements Open access funding provided by University of Vienna.

Open Access This article is distributed under the terms of the Creative Commons Attribution 4.0 International License (http://creativecommons.org/licenses/by/4.0/), which permits unrestricted use, distribution, and reproduction in any medium, provided you give appropriate credit to the original author(s) and the source, provide a link to the Creative Commons license, and indicate if changes were made.

\section{References}

Bultmann R, Caulkins JP, Feichtinger G, Tragler G (2008) How should policy respond to disruptions in markets for illegal drugs? Contemp Drug Probl 35(2-3):371-395

Caulkins J, Feichtinger G, Grass D, Hartl RF, Kort PM, Seidl A (2013a) When to make proprietary software open source. J Econ Dyn Control 37(6):1182-1194. https://doi.org/10.1016/j.jedc.2013.02.009

Caulkins JP, Hartl RF, Kort PM, Feichtinger G (2007) Explaining fashion cycles: Imitators chasing innovators in product space. J Econ Dyn Control 31(5):1535-1556

Caulkins JP, Feichtinger G, Grass D, Hartl RF, Kort PM (2011a) Two state capital accumulation with heterogenous products: disruptive vs. non-disruptive goods. J Econ Dyn Control 35(4):462-478

Caulkins JP, Feichtinger G, Grass D, Hartl RF, Kort PM, Seidl A (2011b) Optimal pricing of a conspicuous product during a recession that freezes capital markets. J Econ Dyn Control 35(1):163-174. https:// doi.org/10.1016/j.jedc.2010.09.001

Caulkins JP, Feichtinger G, Hartl RF, Kort PM, Novak AJ, Seidl A (2013b) Long term implications of drug policy shifts: anticipating and non-anticipating consumers. Ann Rev Control 37(1):105-115

Caulkins JP, Feichtinger G, Grass D, Hartl RF, Kort PM, Seidl A (2015) Capital stock management during a recession that freezes credit markets. J Econ Behav Organ 116:1-14. https://doi.org/10.1016/j.jebo. 2015.02 .023

Chahim M, Hartl RF, Kort PM (2012) A tutorial on the deterministic impulse control maximum principle: necessary and sufficient optimality conditions. Eur J Oper Res 219(1):18-26

Coase RH (1972) Durability and monopoly. J Law Econ 15(1):143-149

Dhebar A (1994) Durable-goods monopolists, rational consumers, and improving products. Mark Sci 13(1):100-120 
Feichtinger G, Hartl RF, Kort PM, Veliov VM (2004) Dynamic investment behavior taking into account ageing of the capital good. In: Udwadia FE, Weber HI, Leitmann G (eds) Dynamical systems and control. CRC, Boca Raton, pp 379-391

Feichtinger G, Hartl RF, Kort PM, Veliov VM (2006) Anticipation effects of technological progress on capital accumulation: a vintage capital approach. J Econ Theory 126(1):143-164

Grass D, Caulkins JP, Feichtinger G, Tragler G, Behrens DA (2008) Optimal control of nonlinear processes: with applications in drugs, corruption and terror. Springer, Heidelberg

Hartl RF (1987) A simple proof of the monotonicity of the state trajectories in autonomous control problems. J Econ Theory 40(1):211-215

Ho TH, Savin S, Terwiesch C (2002) Managing demand and sales dynamics in new product diffusion under supply constraint. Manag Sci 48(2):187-206

Ho TH, Savin S, Terwiesch C (2011) Note: A reply to "new product diffusion decisions under supply constraints". Manag Sci 57(10):1811-1812

Kornish LJ (2001) Pricing for a durable-goods monopolist under rapid sequential innovation. Manag Sci 47(11):1552-1561

Li H, Graves SC (2012) Pricing decisions during inter-generational product transition. Prod Oper Manag 21(1):14-28

McAfee RP, Wiseman T (2008) Capacity choice counters the Coase conjecture. Rev Econ Stud 75(1):317331

Montez J (2013) Inefficient sales delays by a durable-good monopoly facing a finite number of buyers. Rand J Econ 44(3):425-437

Moser E, Seidl A, Feichtinger G (2014) History-dependence in production-pollution-trade-off models: a multi-stage approach. Ann Oper Res 222(1):457-481

Seidl A (2012) Periodic updates relying on open source software: an impulse approach. IFAC Proc Vol 45(25):7-11

Seidl A, Hartl RF, Kort PM (2019) A multi-stage optimal control approach of durable goods pricing and the launch of new product generations. Automatica 106:207-220

Şeref MMH, Carrillo JE, Yenipazarli A (2016) Multi-generation pricing and timing decisions in new product development. Int J Prod Res 54(7):1919-1937

Sethi SP, Thompson GL (2000) Optimal control theory applications to management science and economics, 2nd edn. Kluwer Academic Publishers, Boston

Shen W, Duenyas I, Kapuscinski R (2014) Optimal pricing, production, and inventory for new product diffusion under supply constraints. Manuf Serv Oper Manag 16(1):28-45

Tirole J (1988) The theory of industrial organization. MIT Press, Cambridge

Publisher's Note Springer Nature remains neutral with regard to jurisdictional claims in published maps and institutional affiliations. 